\title{
Quadratus Lumborum Block: a Technical Review
}

\author{
Avni Gupta $^{1} \cdot$ Rakesh Sondekoppam ${ }^{2} \cdot$ Hari Kalagara $^{3}$ \\ Published online: 22 June 2019 \\ (C) The Author(s) 2019, corrected publication November 2019
}

\begin{abstract}
Purpose of Review Ultrasound-guided quadratus lumborum block (QLB) is gaining popularity in regional anesthesia for various surgical procedures. The purpose of this review is to understand the relevant clinical anatomy, different mechanisms of actions, and techniques used for the block and clinical evidence so far.

Recent Findings The current data suggests a wide dermatomal distribution of the local anesthetic from T7-L2. The evidence regarding its utility is still evolving but has shown reduced opioid requirements for cesarean sections, lower abdominal surgery, and hip surgery. Prolonged analgesia has been reported as compared with more conventional transversus abdominis plane (TAP) block. This block has also been reported for above knee amputation, femoral-pop bypass, lumbar laminectomy and fusion, iliac crest bone graft, and iliac and acetabulum fracture.

Summary Quadratus lumborum can be performed through different approaches which requires sound knowledge of anatomy. Further research to determine which approach yields best results is warranted.
\end{abstract}

Keywords Quadratus lumborum block $\cdot$ Ultrasound $\cdot$ Regional anesthesia $\cdot$ Cesarean section $\cdot$ Abdominal procedures $\cdot$ Hip surgery

\section{Introduction}

Quadratus lumborum block (QLB) under ultrasound has been one of the interfascial plane blocks being popularized in regional anesthesia over the last few years given the vast number of indications in a variety of abdomino-pelvic surgeries in pediatrics and adults. In clinical studies, it also has been shown to have opioid sparing effects [1-5] and prolonged post-operative analgesia than more conventional procedures like TAP blocks $[6 \bullet \bullet$. The current review focuses on the anatomical considerations, different approaches, and a review of the safety and clinical efficacy of this block. Differences in technique may result in a differential spread of local anesthetic leading to variation in sensory and motor dermatomal

This article is part of the Topical Collection on Regional Anesthesia

Hari Kalagara

hkalagara@uabmc.edu

1 Millennium Pain Center, Unity Point Health-Methodist Hospital, Peoria, IL, USA

2 University of Alberta Hospital, Edmonton, Alberta, Canada

3 Department of Anesthesiology, The University of Alabama at Birmingham (UAB), Birmingham, AL, USA blockade [7-9]. Further research regarding the best technique to do this block is warranted.

\section{Anatomical Considerations}

Quadratus lumborum muscle (QLM) is an axial muscle situated in the deep posterior abdominal wall mainly intended to stabilize the spine and also acts as an accessory muscle of inspiration. Its function is to stabilize the thorax during respiration and hence has its origin from the inner lip of the posteriomedial iliac crest and inserts into the transverse process of L1-4 as well as medial border of the 12th rib. Psoas major lies anterior to quadratus lumborum on either side of the vertebral body. Posterior to quadratus lumborum lies the group of muscles called erector spinae consisting of multifidus, longissimus, and iliocostalis. Lateral and posterior to quadratus lumborum lies the anterior abdominal wall muscle group, namely, transversus abdominis, internal oblique, and external oblique from anterior to posterior (Fig. 1). Lateral and anterior to quadratus lumborum lay the retroperitoneal structures like the kidney, paranephric fat, posterior renal fascia, and anterior thoracolumbar fascia/transversalis fascia. Both quadratus lumborum and psoas muscle lie 


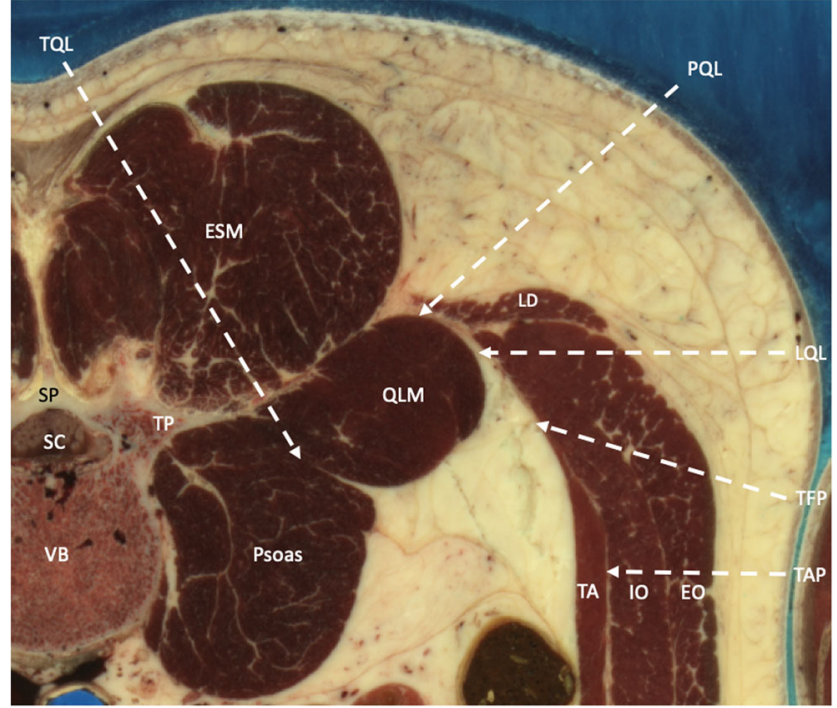

Fig. 1 Cross-section of the posterior abdominal wall at L4 vertebral level showing the relationship of quadratus lumborum muscle (QLM) to the transverse process (TP), erector spinae group of muscles (ESM), and the latissimus dorsi muscle (LD). Spinal cord (SC) is also shown along with the three abdominal muscles: the transversus abdominis (TA), internal oblique (IO), and external oblique (EO) muscles. The various approaches to QLB [lateral QLB (LQL), anterior/transmuscular QLB (TQL), and posterior QLB (PQL)] are shown in dashed arrows along with transversus abdominis plane block (TAP) and the transversalis fascia plane block (TFP). Courtesy Prof. R.D. Hersch, Ecole Polytechnique Fédérale de Lausanne (EPFL), Switzerland. Picture from the former Visible Human Web Server

posterior to the lateral and medial arcuate ligament of the diaphragm respectively.

Quadratus lumborum is surrounded by thoracolumbar fascia. Posterior thoracolumbar fascia surrounds the erector spinae muscle. Middle thoracolumbar fascia lies between the erector spinae and quadratus lumborum, whereas the anterior layer lies anterior to quadratus lumborum and psoas major [10]. Anterior thoracolumbar fascia is continuous with the transversalis fascia, the deep fascia of the abdomen in a twolayer model. The anatomy of the anterior thoracolumbar fascia/transversalis fascia is unique and may be crucial to our understanding of the pattern of spread following injections ventral to the QLM. Transversalis fascia divides into two layers: the inner layer is continuous with endothoracic fascia in the thorax and the outer layer blends with the arcuate ligaments of the diaphragm. Endothoracic fascia provides a potential pathway for cephalad spread of local anesthetic from the abdomen to the thoracic paravertebral space $[7,11,12]$. While there is a channel for the injectate deposited on the ventral aspect of the QLM to reach the thoracic paravertebral space, whether this is clinically relevant needs further studies. Another important anatomical consideration is that the psoas major muscle lies in close proximity to QLM and is ventromedially located. The psoas major muscle while housing the lumbo-sacral plexus may be commonly split by a fascial layer between the posterior $1 / 3$ and anterior $2 / 3$ of the muscle thereby allowing a continuity with the transversalis fascia over the ventral aspect of the quadratus lumborum. This may be a potential path of local anesthetic spread from QLB to the lumbar plexus.

\section{Neurovasculature}

The iliohypogastric, ilioinguinal, and subcostal nerves lay on the ventral aspect of quadratus lumborum muscle and are encased within the transversalis fascia [13]. The consistent level of sensory dermatomal level achieved through quadratus lumborum block involves T12-L2 with various techniques [14-21]. This explains the consistent blockade of these three nerves since these nerves travel over the QLM and are directly related to the muscle $[7,9 \bullet, 14-22]$. The lateral femoral cutaneous nerve, obturator, and femoral nerve lie within the psoas muscle at L4 or L5 vertebral level and exit the muscle more caudally $[23,24]$. The data does not show consistent blockade of these nerves but there is a potential for the spread as discussed above. Posterior to quadratus lumborum are the dorsal rami of spinal nerves innervating the erector spinae muscles. Sympathetic nerve fibers innervating abdominal muscles are also located posterior to quadratus lumborum and innervate thoracolumbar fascia [25]. This could be one of the reasons for analgesia provided through the posterior approach of quadratus lumborum block [26]. Lumbar arteries travel in close proximity and sometimes within the substance of the QLM. They may be a source of bleeding following the block and we recommend color doppler query in the path of needle trajectory before performing the block.

\section{Technical Considerations}

Although there is some controversy regarding nomenclature of different approaches to perform the block, the most popular consensus is to name on the basis of anatomical location of needle tip in relation to quadratus lumborum muscle [27••]. Hence, the three different approaches that can be easily remembered are with respect to the QLM itself which are the lateral, posterior, and anterior quadratus lumborum block.

Standard safety and aseptic measures prior to performing for all regional blocks should be followed. The patient can be positioned in prone, lateral, or sitting position depending on patient and physician preferences. We recommend in-plane ultrasound approach with direct needle visualization along with hydro dissection using a low-frequency curvilinear probe given that it is a deeper block. Typical needle length used is $80-150 \mathrm{~mm}$ depending on body habitus of the patient. The block can be done as a single shot injection as well as a continuous catheter infusion, which determines gauge of the needle. The most common local anesthetics used are $0.2-0.5 \%$ 
Fig. 2 Lateral QLB without (a) and with (b) highlighting. QL, quadratus lumborum muscle; TA, transversus abdominis tapering into transversalis fascia at the lateral border of QL; IO, internal oblique muscle; EO, external oblique muscle. The needle is shown in yellow arrow
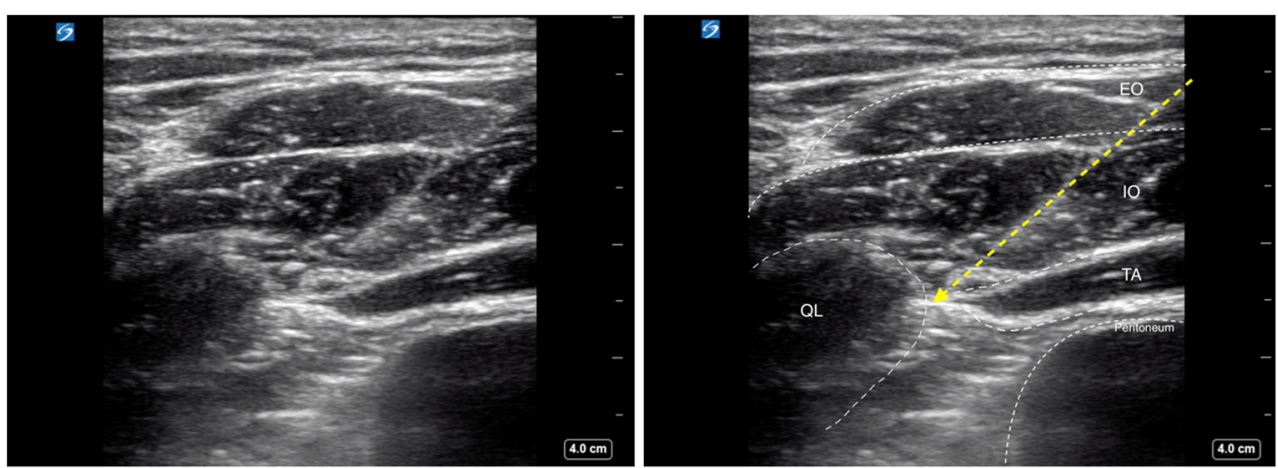

ropivacaine $[2 \bullet, 3]$ or $0.1-0.25 \%$ bupivacaine $[1,5 \bullet \bullet, 6 \bullet \bullet$. Due to large volumes injected, special attention should be made regarding toxic threshold of local anesthetic selected for blockade. Typical volume used ranges from $0.2-0.5 \mathrm{ml} /$ $\mathrm{kg}$ (ref) on each side [1-6].

The figure demonstrates different approaches of block in relation to anatomy (Fig. 1). There is insufficient evidence recommending one approach over the other for specific surgical indications.

For lateral quadratus lumborum block (Fig. 2), the needle is positioned lateral to the ultrasound probe in the anterior to posterior trajectory. The needle further penetrates the anterior abdominal wall muscle group (external oblique, internal oblique, and transversus abdominis). The final position of the needle is lateral to quadratus lumborum. The injectate spreads to transversus abdominis muscle plane and subcutaneous area [28 ], although clinically, a more extensive distribution has been reported [2-4].

The needle trajectory can be anterior-posterior or posterioranterior for posterior quadratus lumborum block. The final position of the needle tip is between erector spinae and quadratus lumborum muscle on the posterior surface of quadratus lumborum muscle (Fig. 3). This approach demonstrates the spread along middle thoracolumbar fascia $[9 \bullet, 28 \bullet]$.

Similar to the posterior quadratus lumborum, the anterior quadratus lumborum can have either an anterior-posterior or posterior-anterior needle trajectory [29••, 30] with the final position of the needle tip anterior to the quadratus lumborum

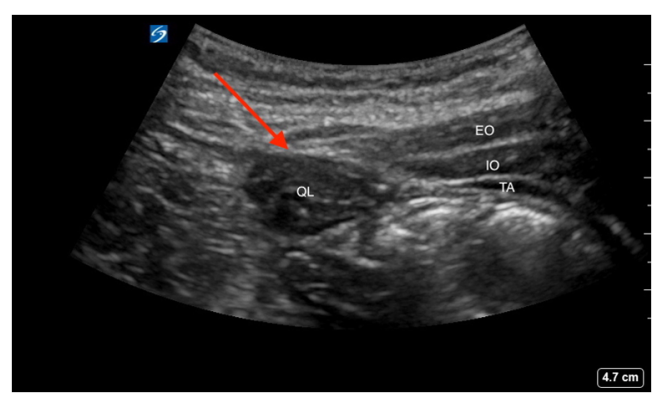

Fig. 3 Posterior QLB. QL, quadratus lumborum muscle; EO, external oblique muscle; IO, internal oblique muscle; TA, transversus abdominis muscle; red arrow, needle path for posterior QLB muscle between quadratus lumborum and psoas major (Fig. 4). Alternatively, a subcostal oblique approach has been described in which the probe is placed in the parasagittal plane tilted medially at the level of the 12th rib approximately 4 $6 \mathrm{~cm}$ from the midline [8]. The needle is introduced caudal to the transducer probe, in-plane in a caudal to cranial trajectory with the final position as mentioned above (Fig. 5). The spread has been along the anterior thoracolumbar fascia through the endothoracic fascia into the thoracic paravertebral space in addition to blocking the lumbar nerve roots [7, 9•, 22].

\section{Indications and Clinical Evidence}

Based on the current randomized trials and case reports, quadratus lumborum blocks have been used for multiple surgeries, including cesarean section $[1,2,3 \bullet \bullet, 6 \bullet \bullet$ and gynecological [31] and lower abdominal surgeries [5••, 32]. It has also been used for analgesia for colostomy closure [16], hernia repairs [33, 34], gastrectomy [35], and nephrectomy [17, 36-39]. It has been used for various lower limb surgeries like hip arthroplasty [4, 14, 18, 20, 40-42], above knee amputation in combination with sciatic block [43], iliac crest bone graft, and iliac and acetabulum fracture [44]. It has been used in conjunction with lumbar plexus block and sciatic nerve blocks for femoral-popliteal bypass [45]. There has been a case report regarding the utilization of this block of lumbar laminectomy and fusion [46]. The use of posterior quadratus lumborum

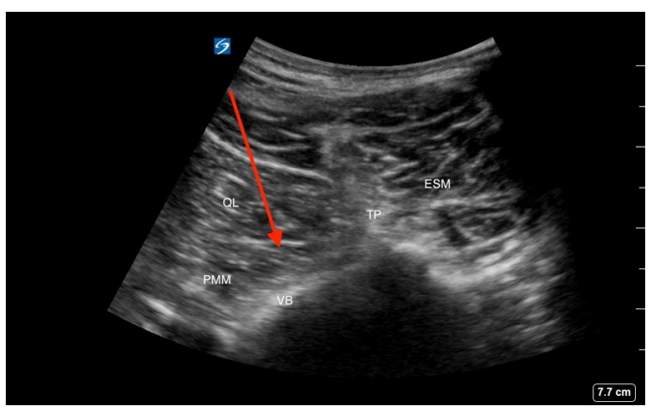

Fig. 4 Anterior QLB. TP, transverse process; VB, vertebral body; QL, quadratus lumborum muscle; ESM, erector spinae muscle; PMM, psoas major muscle; red arrow, needle path for anterior QLB 


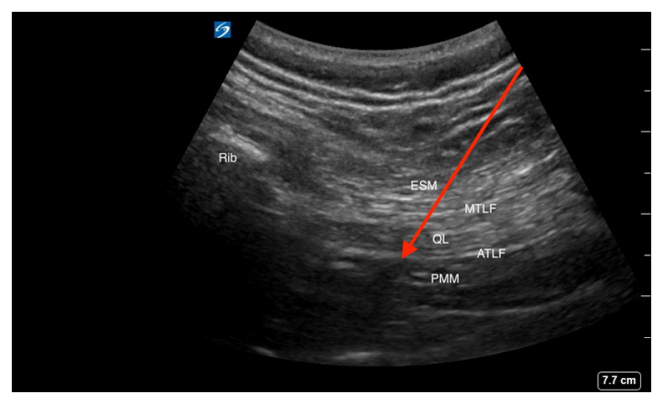

Fig. 5 Subcostal QLB. Rib, 12th rib; ESM, erector spinae muscle; QL, quadratus lumborum muscle; PMM, psoas major muscle; MTLF, middle thoracolumbar fascia; ATLF, anterior thoracolumbar fascia; red arrow, needle path for anterior subcostal QLB

block for breast reconstruction using transverse rectus abdominis flaps has also been reported [15].

Posterior quadratus lumborum block has been shown to reduce morphine requirements for $48 \mathrm{~h}$ as compared with placebo and TAP block after cesarean sections in two randomized control trials $[1,6 \cdot \bullet]$. Two further randomized control trials using lateral quadratus lumborum block also showed reduced opiate consumption after cesarean sections $[2 \cdot 3]$. Lateral quadratus lumborum block has been shown to reduce Visual Analogue Scale (VAS) pain score and 24-h opioid consumption as compared with femoral nerve block for hip hemiarthroplasty for femoral nerve fracture in another randomized control trial [4]. Reduced Numeric Rating Scale (NRS) pain scores have been shown in laparoscopic gynecological surgery with posterior quadratus lumborum block [30]. A randomized control trial done in the pediatric age group for inguinal hernia surgery/orchiopexy showed reduced rescue analgesia requirements using posterior quadratus lumborum block as compared with TAP block [5••]. Transmuscular quadratus lumborum block used in patients undergoing total hip arthroplasty showed reduction in length of stay and intraoperative opioid use [42].

\section{Contraindications}

Allergy to local anesthetics, local site infection, sepsis, bleeding disorder, and anticoagulation are absolute contraindications for the procedure since it is a deep block. Relative contraindications include known neurological disorder, anatomic abnormalities, and hemodynamically unstable patient.

\section{Complications}

Lower extremity weakness has been reported with quadratus lumborum block leading to delay in mobilization and prolonged hospital stay $[47,48]$. Local anesthetic distribution to the nerve roots or branches of lumbar plexus through spread in paravertebral spaces or via transversalis fascia is likely responsible for weakness of hip flexors (psoas and iliacus) and knee extensors (quadriceps). Quadriceps weakness was reported to be most commonly associated with anterior quadratus lumborum block, followed by posterior and lateral approaches respectively [47]. There is insufficient data on the recommended approach of the block as well as concentration or volume of local anesthetic to be used to avoid this complication.

Hypotension has been reported and is possibly related to spread of local anesthetic in the paravertebral spaces [35]. Other considerations should be local anesthetic toxicity due to large volume used specially in cases of bilateral blocks [49].

The complications related to technical challenges of the deeper block and inadequate visualization and hence injury to surrounding structures should be kept in mind. Injury to pleura, kidney, retroperitoneal hematoma, and nerve roots are potential complications. Full aseptic precautions should be used to avoid infection or abscess formation.

\section{Caveats}

- Interfascial blocks have variability in injectate spread. This is also true for QLB as evident from both clinical and anatomical studies. Thus, an injectate following QLB definitely covers the T12-L1 nerves as they travel in direct relation to the muscle itself but depending on the surrounding anatomy and injectate pressure, it can spread cephalad to the lower thoracic paravertebral space, laterally into the TAP plane, anteriorly into the psoas major muscle, and thus to the lumbar plexus.

- Anatomical studies and case reports are only hypothesis generating and should not be taken as clinical evidence. Hence, clinical decision-making should not be based on their findings.

- There is still a need for better evidence with regard to the best approach and the utility of the technique itself in the form of multicenter randomized controlled trials (RCT) and in comparison with the current gold standard techniques such as thoracic epidurals for abdominal surgeries and probably peripheral nerve blocks for lower extremity surgeries.

- QLB are deep blocks and hence may offset the presumed safety advantages of other interfascial plane blocks such as TAP blocks and rectus sheath blocks. We are still unfamiliar with the clinical presentation of hematological complications and based on the limited evidence, it seems that the hematological complications may have a delayed and atypical presentation. 


\section{Conclusions}

There is upcoming clinical evidence that quadratus lumborum block can provide perioperative analgesia for various lower abdominal, pelvic, and hip surgeries due to a consistent dermatomal coverage of the surgical site. While it has also been used for upper abdominal, renal, and vascular surgeries, further evidence is warranted. Different approaches have been described to perform the block requiring a sound knowledge of anatomy and technical expertise of ultrasound. The data, although limited to few studies, shows prolonged analgesia, reduced hospital stay, and reduced opioid requirements for abdominal surgeries, cesarean section, and hip surgery.

\section{Compliance with Ethical Standards}

Conflict of Interest Avni Gupta, Rakesh Sondekoppam, and Hari Kalagara declare they have no conflict of interest.

Human and Animal Rights and Informed Consent This article does not contain any studies with human or animal subjects performed by any of the authors.

Open Access This article is licensed under a Creative Commons Attribution 4.0 International License (https://creativecommons.org/licenses/ by/4.0/), which permits use, sharing, adaptation, distribution and reproduction in any medium or format, as long as you give appropriate credit to the original author(s) and the source, provide a link to the Creative Commons licence, and indicate if changes were made. The images or other third party material in this article are included in the article's Creative Commons licence, unless indicated otherwise in a credit line to the material. If material is not included in the article's Creative Commons licence and your intended use is not permitted by statutory regulation or exceeds the permitted use, you will need to obtain permission directly from the copyright holder. To view a copy of this licence, visit http://creativecommons.org/licenses/by/4.0/.

\section{References}

Papers of particular interest, published recently, have been highlighted as:

- Of importance

•. Of major importance

1. Blanco R, Ansari T, Girgis E. Quadratus lumborum block for postoperative pain after caesarean section: a randomised controlled trial. Eur J Anaesthesiol. 2015;32:812-8.

2. Krohg A, Ullensvang K, Rosseland LA, Langesæter E, Sauter AR. The analgesic effect of ultrasound-guided quadratus lumborum block after cesarean delivery: a randomized clinical trial. Anesth Analg. 2018;126:559-65 RCT showing the analgesic benefit of ultrasound-guided posterior quadratus lumborum block with ropivacaine versus saline for post cesarean delivery opioid consumption and pain intensity scores.

3. Mieszkowski MM, Mayzner-Zawadzka E, Tuyakov B, Mieszkowska M, Żukowski M, Waśniewski T, et al. Evaluation of the effectiveness of the quadratus lumborum block type I using ropivacaine in postoperative analgesia after a cesarean section: a controlled clinical study. Ginekol Pol. 2018;89:89-96.

4. Parras T, Blanco R. Randomised trial comparing the transversus abdominis plane block posterior approach or quadratus lumborum block type I with femoral block for postoperative analgesia in femoral neck fracture, both ultrasound-guided. Rev Esp Anestesiol Reanim. 2016;63:141-8.

5.• Oksüz G, Bilal B, Gürkan Y, Urfalioğlu A, Arslan M, Giși G, et al. Quadratus lumborum block versus transversus abdominis plane block in children undergoing low abdominal surgery: a randomized controlled trial. Reg Anesth Pain Med. 2017;42:674-9 Prospective, double-blind, randomized study in pediatric patients undergoing unilateral inguinal hernia repair or orchiopexy. Quadratus lumborum block provided superior and prolonged analgesia compared to Transversus abdominis plane block.

6.• Blanco R, Ansari T, Riad W, Shetty N. Quadratus lumborum block versus transversus abdominis plane block for postoperative pain after cesarean delivery: a randomized controlled trial. Reg Anesth Pain Med. 2016;41:757-62 A RCT trial comparing the quadratus lumborum block versus Transversus abdominis plane block for postoperative pain after cesarean delivery. This study showed the benefits of QL block over TAP block in reducing the morphine consumption up to 48 hours postoperatively.

7. Dam M, Moriggl B, Hansen CK, Hoermann R, Bendtsen TF, Børglum J. The pathway of injectate spread with the transmuscular quadratus lumborum block: a cadaver study. Anesth Analg. 2017;125:303-12.

8. Elsharkawy, H, Ahuja, S, DeGrande, S, Maheshwari, K, Chan, V (2019) Subcostal approach to anterior quadratus lumborum block for pain control following open urological procedures. J. Anesth. (2019) 33:148.

9. Elsharkawy H, El-Boghdadly K, Kolli S, Esa WA, DeGrande S, Soliman LM, et al. Injectate spread following anterior sub-costal and posterior approaches to the quadratus lumborum block. Eur J Anaesthesiol. 2017;34:587-95 A comparative cadaveric study demonstrating the injectate spread following posterior and anterior quadratus lumborum blocks. This study demonstrated various nerves and thoracolumbar fascia involved with posterior and anterior QL blocks.

10. Willard FH, Vleeming A, Schuenke MD, Danneels L, Schleip R. The thoracolumbar fascia: anatomy, function and clinical considerations. J Anat. 2012;221:507-36.

11. Saito T, Den S, Tanuma K, Tanuma Y, Carney E, Carlsson C. Anatomical bases for paravertebral anesthetic block: fluid communication between the thoracic and lumbar paravertebral regions. Surg Radiol Anat. 1999;21:359-63.

12. Karmakar MK, Gin T, Ho AM. Ipsilateral thoraco-lumbar anaesthesia and paravertebral spread after low thoracic paravertebral injection. Br J Anaesth. 2001;87:312-6.

13. Klaassen Z, Marshall E, Tubbs RS, Louis RG Jr, Wartmann CT, Loukas M. Anatomy of the ilioinguinal and iliohypogastric nerves with observations of their spinal nerve contributions. Clin Anat. 2011;24:454-61.

14. La Colla L, Ben-David B, Merman R. Quadratus lumborum block as an alternative to lumbar plexus block for hip surgery: a report of 2 cases. A A Case Rep. 2017;8:4-6.

15. Spence NZ, Olszynski P, Lehan A, Horn JL, Webb CA. Quadratus lumborum catheters for breast reconstruction requiring transverse rectus abdominis myocutaneous flaps. J Anesth. 2016;30:506-9.

16. Visoiu M, Yakovleva N. Continuous postoperative analgesia via quadratus lumborum block: an alternative to transversus abdominis plane block. Paediatr Anaesth. 2013;23:959-61. 
17. Chakraborty A, Goswami J, Patro V. Ultrasound-guided continuous quadratus lumborum block for postoperative analgesia in a pediatric patient. A A Case Rep. 2015;4:34-6.

18. Ueshima H, Yoshiyama S, Otake H. The ultrasound-guided continuous transmuscular quadratus lumborum block is an effective analgesia for total hip arthroplasty. J Clin Anesth. 2016;31:35.

19. Kadam VR. Ultrasound-guided quadratus lumborum block as a postoperative analgesic technique for laparotomy. J Anaesthesiol Clin Pharmacol. 2013;29:550-2.

20. Johnston DF, Sondekoppam RV. Continuous quadratus lumborum block analgesia for total hip arthroplasty revision. J Clin Anesth. 2016;35:235-7.

21. Hernandez MA, Vecchione T, Boretsky K. Dermatomal spread following posterior transversus abdominis plane block in pediatric patients: our initial experience. Paediatr Anaesth. 2017;27:300-4.

22. Sondekoppam RV, Ip V, Johnston DF, Uppal V, Johnson M, Ganapathy S, et al. Ultrasound-guided lateral-medial transmuscular quadratus lumborum block for analgesia following anterior iliac crest bone graft harvesting: a clinical and anatomical study. Can J Anaesth. 2018;65:178-87.

23. Dietemann JL, Sick H, Wolfram-Gabel R, Cruz da Silva R, Koritke JG, Wackenheim A. Anatomy and computed tomography of the normal lumbosacral plexus. Neuroradiology. 1987;29:58-68.

24. Awad IT, Duggan EM. Posterior lumbar plexus block: anatomy, approaches, and techniques. Reg Anesth Pain Med. 2005;30:143-9.

25. Benetazzo L, Bizzego A, De Caro R, Frigo G, Guidolin D, Stecco C. 3D reconstruction of the crural and thoracolumbar fasciae. Surg Radiol Anat. 2011;33:855-62.

26. Arnér S, Lindblom U, Meyerson BA, Molander C. Prolonged relief of neuralgia after regional anesthetic blocks: a call for further experimental and systematic clinical studies. Pain. 1990;43:287-97.

27.• El-Boghdadly K, Elsharkawy H, Short A, Chin KJ. Quadratus lumborum block nomenclature and anatomical considerations. Reg Anesth Pain Med. 2016;41:548-9 Letter to the editor describing the Quadratus lumborum block nomenclature based on the anatomical considerations. This describes the lateral QL, posterior QL and anterior QL blocks along with various thoracolumbar fascia.

28. Carline L, McLeod GA, Lamb C. A cadaver study comparing spread of dye and nerve involvement after three different quadratus lumborum blocks. Br J Anaesth. 2016;117:387-94 A cadaver study comparing the dye spread following QL1, QL2 and Transmuscular QL blocks. This cadaver study demonstrates the muscles and nerves involved with 3 different types of QL blocks using the old nomenclature.

29.• Dam M, Hansen CK, Børglum J, Chan V, Bendtsen TF. A transverse oblique approach to the transmuscular quadratus lumborum block. Anaesthesia. 2016;71:603-4 A technical description of Transmuscular quadratus lumborum block and the Shamrock sign.

30. Elsharkawy H. Quadratus lumborum block with paramedian sagittal oblique (subcostal) approach. Anaesthesia. 2016;71:241-2.

31. Ishio J, Komasawa N, Kido H, Minami T. Evaluation of ultrasoundguided posterior quadratus lumborum block for postoperative analgesia after laparoscopic gynecologic surgery. J Clin Anesth. 2017;41:1-4.

32. Shaaban, M, Esa, WA, Maheshwari, K, Elsharkawy, H, Soliman, LM Bilateral continuous quadratus lumborum block for acute postoperative abdominal pain as a rescue after opioid-induced respiratory depression. A A Case Rep. 2015 Oct 1;5(7):107-11.
33. Aksu C, Gürkan Y. Ultrasound guided quadratus lumborum block for postoperative analgesia in pediatric ambulatory inguinal hernia repair. J Clin Anesth. 2018;46:77-8.

34. Carvalho R, Segura E, Loureiro MD, Assunção JP. Quadratus lumborum block in chronic pain after abdominal hernia repair: case report. [Article in Portuguese.]. Rev Bras Anestesiol. 2017;67:107-9.

35. Sá M, Cardoso JM, Reis H, Esteves M, Sampaio J, Gouveia I, et al. Quadratus lumborum block: are we aware of its side effects? A report of 2 cases. [Article in Portuguese. Rev Bras Anestesiol. 2017;68:396-9.

36. Corso RM, Piraccini E, Sorbello M, Bellantonio D, Tedesco M. Ultrasound-guided transmuscular quadratus lumborum block for perioperative analgesia in open nephrectomy. Minerva Anestesiol. 2017;83:1334-5.

37. Suri A, Sindwani G, Sahu S, Gupta N, Sureka S. Surgeon assisted quadratus lumborum block: "Gaurav-Aditi" technique case series. J Clin Anesth. 2017;43:48-9.

38. Ueshima H, Otake H. Clinical experiences of unilateral anterior sub-costal quadratus lumborum block for a nephrectomy. J Clin Anesth. 2018;44:120.

39. Sindwani G, Suri A, Shrivastava D, Sureka S. Laparoscopic guided continuous type 1 quadratus lumborum block: Sindwani technique with case series. J Clin Anesth. 2017;42:93-4.

40. Hockett MM, Hembrador S, Lee A. Continuous quadratus lumborum block for postoperative pain in total hip arthroplasty: a case report. A A Case Rep. 2016;7:129-31.

41. Ohgoshi Y, Nakayama H, Kubo EN, Izawa H, Kori S, Matsukawa M. Clinical experiences of the continuous quadratus lumborum block via paramedian sagittal oblique approach. J Clin Anesth. 2017;38:89-90.

42. Green M, Hoffman CR, Iqbal U, Ives OO, Hurd B. Transmuscular quadratus lumborum block reduced length of stay in patients receiving total hip arthroplasty. Anesth Pain Med. 2018;8(6):e80233.

43. Ueshima $\mathrm{H}$, Otake $\mathrm{H}$. Lower limb amputations performed with anterior quadratus lumborum block and sciatic nerve block. J Clin Anesth. 2017;37:145.

44. Segura-Grau E, Magalhães J, Cabral F, Costa C. Continuous quadratus lumborum type 2 block: good analgesia alternative for complex iliac and acetabulum fracture. J Clin Anesth. 2018;46:91.

45. Watanabe K, Mitsuda S, Tokumine J, Lefor AK, Moriyama K, Yorozu T. Quadratus lumborum block for femoral-femoral bypass graft placement: a case report. Medicine (Baltimore). 2016;95:e4437.

46. Iwamitsu R, Ueshima $\mathrm{H}$, Otake $\mathrm{H}$. Intermittent bilateral posterior quadratus lumborum block was effective for pain management in lumbar spinal fusion. J Clin Anesth. 2017;42:16.

47. Ueshima $\mathrm{H}$, Hiroshi O. Incidence of lower-extremity muscle weakness after quadratus lumborum block. J Clin Anesth. 2018;44:104.

48. Wikner M. Unexpected motor weakness following quadratus lumborum block for gynaecological laparoscopy. Anaesthesia. 2017:72:230-2.

49. Gitman M, Barrington MJ. Local anesthetic systemic toxicity: a review of recent case reports and registries. Reg Anesth Pain Med. 2018;43:124-30.

Publisher's Note Springer Nature remains neutral with regard to jurisdictional claims in published maps and institutional affiliations. 